\title{
Modification of Adhesive Joints Resistance with Current Pulses
}

\author{
Pavel Mach, David Bušek \\ Czech Technical University in Prague, Faculty of Electrical Engineering, \\ Department of Electrotechnology Prague, Czech Republic \\ mach@fel.cvut.cz
}

\begin{abstract}
Strips of electrically conductive adhesive were printed on a test PCB. Strips were processed by a standard curing process and then aged at the temperature of $125{ }^{\circ} \mathrm{C}$. One group of samples was aged in an oven; the second group was heated with current pulses of high intensity. Ageing was carried out 150 hours. The frequency, amplitude and pulse duty cycle were changed. The strip resistance was measured by the four-terminal method, the resistance of the contact between strips and pads using the three-terminal method. It was found that the resistance of the strip heated in an oven slowly grew with the time of ageing. The resistance of the samples heated with current pulses first decreased and then started to growth rapidly. The course depended on parameters of the pulses. The reasons of the resistance changes were discussed.
\end{abstract}

\section{INTRODUCTION}

Electrically conductiveadhesives with isotropic electrical conductivity are usually adhesives containing extremely large concentration, typically 70 to $80 \%$ by weight, of electrically conductive filler to give electrical conduction. The filler are silver flakes very often.

Conducting particles are compressed in insulating matrix of electrically conductive adhesive. Different types of resins are used as such the matrix. Epoxy resin it is the most often, but also other types like silicon or polyimide resin are used. Compression is achieved by adhesive curing, which causes its solidification. This process is connected with decrease of the volume of resin. Shrinkage of epoxy resin after curing is $7 \%$ approximately and causes stabilization of contacts among filler particles and creating of conductive net in adhesive [1].

Electrical conductivity of electrically conductive adhesives is significantly lower in comparison with conductivity of solders. There are many ways how to improve this situation. These ways are addition of other conductive particles of proper shapes and dimensions into standard adhesives, intensive rotary stirring before adhesive application, sintering of filler particles and others [2], [3].

Sintering is a very effective method of adhesive conductivity improvement, because is causes welding of the filler particles and elimination of contacts between them. Different types of chemicals or different types of particles added into adhesive, e.g. nanoparticles, are used to support sintering.

Sintering needs higher curing temperature than standard curing process, usually. However the temperature must not be too high, because the main application of electrically conductive adhesives is conductive assembly of components with low temperature resistivity, which does not allow the use of a soldering process.

It was also found that the resistivity of adhesive can be changed with current pulses of high intensity [4]. If short current pulses are applied, adhesive resistivitiy decreases at first, but then it starts to grow. This paper shows the results of experiments when adhesive joints were powered with very short current pulses of high intensity. This topic is important, because it could be one of possible way how to improve the electrical properties of adhesive joints. 


\section{THEORY}

Curing process of adhesives causes significant changes of adhesive properties. First it causes solidification of adhesive, second decrease of its volume (shrinkage of adhesive) and third, with high probability, covering of filler particles with oxides or other types of chemical films. Due to high electrical conductivity of silver oxide such an oxide film does not worse electrical properties of adhesive dramatically, if the filler particles are silver flakes or balls.

Majority types of electrically conductive adhesives used in electronics assembly are based on epoxy resin filled with silver flakes with respect to the price of adhesive. The use of silver is advantage from the point of view of electrical conductivity and conductivity of silver oxide. However, the use of silver is connected with one disadvantage.

Mobility of silver ions is high. Therefore the use of silver in atmosphere with higher humidity can cause migration of silver ions if the electrical voltage is applied between near silver contacts. The result of migration can be a conductive bridge, which will cause short circuit between these contacts. Short connection can damage equipment.

High mobility of silver ions plays an important role in electrically conductive adhesives. It was found that the electrical properties of adhesive can be modified with current pulses of proper intensity and amplitude. Modified can be the resistivity, noise and nonlinearity of $\mathrm{I}-\mathrm{V}$ characteristic of adhesive.

Silver ions under proper conditions migrate in adhesive. The structure of conductive net in adhesive is changed in consequence of such the migration.

Migration of ions can be induced by powering of adhesive with current pulses. If the current pulses are such the amplitude that they do not cause heating of adhesive (heating can cause degradation of resin and covering the filler particles by chemical layers, which cause increase of the contacts resistances between them), that means when amplitudes of pulses are low, changes of electrical properties are caused with ions migration only. If amplitudes of pulses are higher, resin degradation contributes to the changes as well .

It is possible to observe a following course of resistance changes of adhesive. If the frequency of the current pulses has a proper value and pulses have proper amplitude, conductivity of the joints increases. After some time it achieves a maximum value and then starts to decrease. This break can be explained as follows: in the first phase migration of silver ions causes forming of new conductive bridges in adhesive. These bridges increase density of conductive net and improve adhesive conductivity. When the energy supplied into adhesive is too high, heating of adhesive causes releasing of chemical groups of adhesive, which react with the particles of filler. The particles are covered with chemical layers, which increase the contact resistance. Conductivity of contacts between particles decreases.

The goal of this work was to test if temperature ageing of adhesive joints in an oven and their ageing using current pulses on the same temperature will cause the comparable changes of the joints resistance. If the changes will be near, no ion migration appeared in adhesive. If changes will differ, it is possible to think over about ion migration in adhesive, which was caused by current pulses and which takes part on these changes.

\section{EXPERIMENT}

Ag filled bisphenol epoxy resin (AX20, Amepox) was used for fabrication of test samples (see Fig. 1) by stencil printing. The thickness of the film was 300 microns before curing.

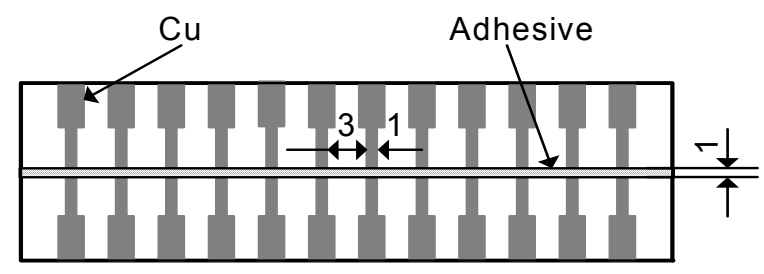

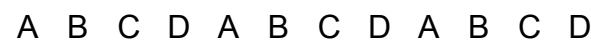

Fig. 1. Samples for measurement

A pulse generator designed and fabricated at the Department of Electrotechnology was used for loading of the joints with the current pulses. The pulse duty factor was adjustable, the possible highest amplitude of the pulses was $10 \mathrm{~A}$, the frequency of the pulses was changed in the range of $10 \mathrm{~Hz}$ to $1 \mathrm{kHz}$.

The temperature of the adhesive strip heated with pulses was measured using an infrared imaging camera IR EASIR 4. The samples were located at a closed box during the measurement to avoid their 
cooling with agitated air. Calibration was carried out using a Cu-constantan thermocouple. A climatic chamber WTB Binder was used for heating of the samples. The measurements were carried out at the ambient humidity (approx. $74 \% \mathrm{RH}$ ).

Four-terminal and three terminal methods [3] were used for the measurement of the adhesive strip resistance and for the measurement of the contact resistance between adhesive and a $\mathrm{Cu}$ pad (see Fig. 2). The LCR meter HP 4284 was used for the resistance measurement. The measurements were carried after cooling of samples at the normal temperature.

The four-terminal method makes the measurement of the resistance of the strip of adhesive possible without influencing the result with contact resistances. The three-terminal method makes possible the measurement of the contact resistance between adhesive and a pad.

If the switch $\mathrm{S} 1$ is in the position 1, the measurement is carried out in three-terminal configuration. If the switch $\mathrm{S} 1$ is in the position 2, the

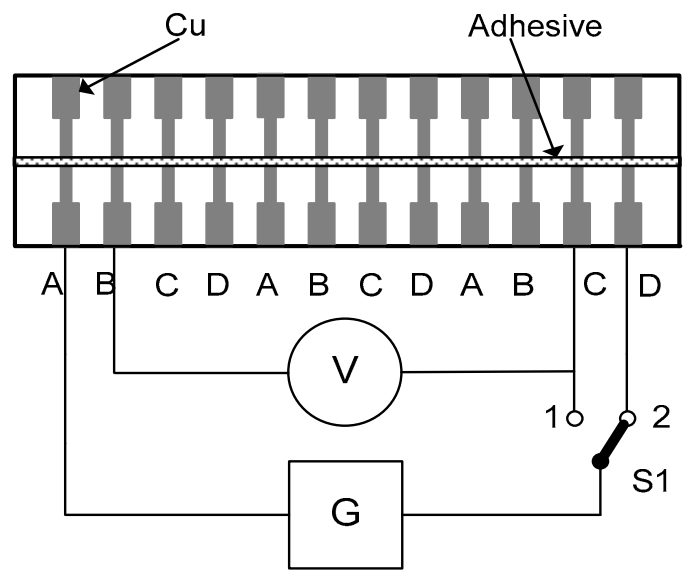

Fig. 2. Measurement using four-terminal and three-terminal method. V - voltmeter, $\mathrm{G}$ - constant current generator. If the switch $\mathrm{S} 1$ is in position 1, the three-terminal

measurement is carried out, if the switch $\mathrm{S} 1$ is in position 2, the four-terminal measurement is carried out [3]

measurement is carried out in four-terminal configuration. The difference of these two measurements (the three- terminal minus the fourterminal) yields the contact resistance.

Calculation of amplitude and width of the pulses was carried out with respect to the maximum accepted increase of temperature of the adhesive joints caused by the pulses. For calculation of heat generated inside adhesive it is assumed that adhesive has similar physical parameters like silver, because concentration of silver particles is $80 \%$ by weight. Specific heat capacity of silver is $232 \mathrm{~J} / \mathrm{kgK}$, density $10500 \mathrm{~kg} / \mathrm{m} 3$. It was calculated that the volume of the adhesive used for fabrication of the strip was $1,5.10^{-8} \mathrm{~m}^{3}$ and the mass of adhesive strip was $0,16 \mathrm{~g}$. Energy necessary for increase of temperature of this volume of adhesive by $1 \mathrm{~K}$ will be $0.06 \mathrm{~J}$. When amplitude of the pulse would be $1 \mathrm{~A}$, its width $1 \mathrm{~ms}$, the strip resistance 0,5 $\Omega$, and the frequency of pulses $500 \mathrm{~Hz}$, then energy, which will cause increase of temperature of adhesive by $1 \mathrm{~K}$, will be supported after $0,25 \mathrm{~s}$. However, actual increase of the temperature will be lower, because adhesive is cooled with the test board, with the heat transfer in surroundings and with heat emission. Therefore the actual conditions of heating of were found experimentally.

The generator consists of a control and a power part. The control part makes control of the pulses frequency, pulse duty factor and amplitude possible. With respect to the possible power of the generator, the output cascade was carefully cooled.

One group of the samples was aged in the oven at the temperature of $125^{\circ} \mathrm{C}$, the second group was heated on the same temperature with the current pulses. Ageing was carried out for 150 hours.

Ageing with the current pulses was carried out for pulse amplitude $3 \mathrm{~A}, 6 \mathrm{~A}$ and $9 \mathrm{~A}$. Frequency of the pulses and pulse duty factor was set up to achieve the temperature of the adhesive strip $125{ }^{\circ} \mathrm{C}$. The lower was pulses amplitude; the higher was the frequency of the pulses.

The resistance of the adhesive strips changed during heating, therefore a feedback between pulses frequency and the requested temperature of the adhesive strips was created. Pulse duty factor was set up, if possible, to the value 1:5. This way it was possible to stabilize the strips temperature in the range of $\pm 6{ }^{\circ} \mathrm{C}$.

\section{ReSUlts}

One example of measured results is shown in Fig. 3. Relative resistance changes differ for heating in an oven and for heating using current pulses. It was found that the higher was the pulses amplitude, the higher was increase of adhesive conductivity at the 
starting phase of ageing, but the higher was the increase of adhesive resistivity at the final phase of ageing.

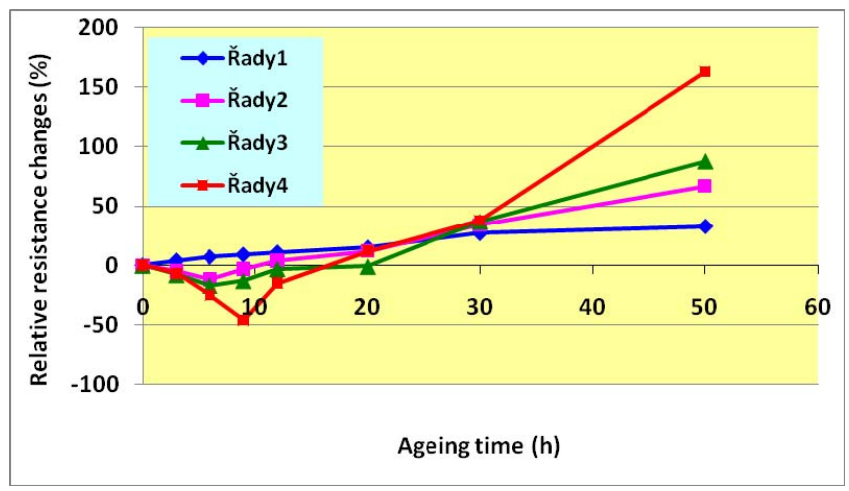

Fig. 3. Relative resistance changes of adhesive strips aged in an oven and by the use of current pulses. Amplitude of pulses $\mathrm{A}$ was $3 \mathrm{~A}$, pulses $\mathrm{B} 6 \mathrm{~A}$ and pulses $\mathrm{C} 9 \mathrm{~A}$

\section{DISCUSSION}

Results of measurements show the strong dependence of the relative resistance changes on the type of heating.

Heating in the oven showed that the adhesive was good cured and that no additional curing occurred during the first phase of ageing. Worsening of electrical conductivity of adhesive was carried out by decrease of quality of contacts between conductive particles. Surprising was that measurements of the contact resistance between adhesive and copper pad of the test board showed very small changes only.

Heating of adhesive using current pulses was very sensitive to adjustment of the pulses parameters. There are many possibilities to arrange the amplitude of the pulses, the frequency and the pulse duty factor. It was interesting, in comparison with the results found for samples aged in an oven that in the starting phase of ageing the conductivity of adhesive increased. This dependence was found for every sample aged with the current pulses. The higher was pulses amplitude, the higher was starting improvement of adhesive conductivity. The reason of such the dependence is breakout of tunnel barriers between conductive particles. With respect to the area of contacts between conductive particles density of the current flowing through the contact is very high and causes sintering of particles with high probability.
This process causes decrease of the contacts resistance and improvement of adhesive conductivity. The higher is the pulses amplitude, the higher is density of the current flowing through the contacts and probability of sintering increases. Therefore the maximum decrease of the resistance was observed for the highest amplitude of pulses.

With the grow of the time of ageing using current pulses, the high temperature of the contact points causes degradation of resin in surroundings of these points. The high temperature can also cause disconnection as result of matrix dilatation and increases probability that they will be covered with chemical products of resin degradation. As a result of this process the resistance of adhesive increases.

\section{Conclusions}

Strips of electrically conductive adhesive with isotropic electrical conductivity were aged at the temperature $125{ }^{\circ} \mathrm{C}$. Heating was carried out in the oven or using current pulses of high intensity. It was found that heating using current pulses cause different changes of the adhesive resistance than heating in the oven due to changes of contact barriers between conductive particles in adhesive.

\section{ACKNOWLEDGEMENT}

The work was carried out as a part of a project "Diagnostics of Materials", number MSM6840770021

\section{REFERENCES}

[1] Su, B.: „Electrical, Thermomechanical and Reliability Modeling of Electrically Conductive Adhesives", Disertation. Georgia Institute of Technology. 2006

[2] Richter, L.: "Study of technologies for support of aggregation of nanoparticles in electrically conductive adhesives". Diploma thesis. CTU Prague, Department of Electrotechnology, 2007

[3] Morris, J. E., Liu, J.: "An Internet Course of conductive adhesives for electronic packaging". Proc. $50^{\text {th }}$ Electronic Components and Technology Conference. Las Vegas, May 2000

[4] Kotthaus, S. et al. Current-Induced Degradation of Isotropically Conductive adhesives, IEEE Trans. on CPMT, Vol. 21, No.2, June 1998, pp.259-265 